\title{
Pacientes em hemodiálise: estado inflamatório e massa magra corporal
}

\author{
Hemodialysis patients: inflammatory status and lean body mass
}

\author{
Annerose Barros ${ }^{1}$, Alex Oliboni Sussela ${ }^{2}$, Raisa Felix ${ }^{2}$, Luiza Silveira Lucas ${ }^{2}$, Domingos O. d'Avila ${ }^{3}$ \\ 1 Nutricionista graduada pelo IPA, Mestre e Doutoranda em Ciências da Saúde/Nefrologia pela Pontifícia Universidade Católica do Rio Grande do Sul (PUCRS). Porto Alegre, RS \\ ${ }^{2}$ Graduandos da Faculdade de Medicina da PUCRS, Porto Alegre, RS; ${ }^{3}$ Médico Nefrologista, Doutor em Nefrologia pela UNIFESP, Professor Titular da Faculdade de \\ Medicina da PUCRS, Professor do Programa de Pós-Graduação em Medicina e Ciências da Saúde da PUCRS, Porto Alegre, RS.
}

Fontes de recursos: Annerose Barros é bolsista de doutorado pelo Conselho Nacional de Desenvolvimento Científico e Tecnológico (CNPq); Alex Oliboni Sussela é bolsista de iniciação científica PIBIC/CNPq; Luiza Silveira Lucas é bolsista de iniciação científica BPA/PUCRS.

\section{RESUMO}

Objetivos: Investigar a relação do estado inflamatório com a massa magra corporal de pacientes em hemodiálise.

Métodos: Um estudo transversal observacional incluiu pacientes em hemodiálise há pelo menos três meses. A análise da composição corporal foi realizada por bioimpedância segmentar multi-frequência (InBody $520^{\circledR}$ ). O estado nutricional associado à inflamação foi avaliado usando o instrumento que computa o Escore Desnutrição-Inflamação.

Resultados: A amostra incluiu 59 indivíduos, sendo 30 mulheres. A idade média foi de 58,7 $\pm 14,4$ anos, a mediana do tempo em hemodiálise foi de 24 (9-49) meses, a média do peso seco estimado foi $67,0 \pm 14,7 \mathrm{~kg}$ e a média de massa magra foi $29,7 \pm 5,5 \mathrm{~kg}$. A mediana do nível sérico de proteína C-reativa ultrassensível foi $8,6(3,9-18,0) \mathrm{mg} / \mathrm{L}$ e acima do limite normal $(\leq 5,0 \mathrm{mg} / \mathrm{L})$, sugerindo a presença de inflamação. $\mathrm{O}$ escore desnutrição-inflamação teve mediana de 4 (2-6). Houve correlação significativa entre o escore desnutrição-inflamação e a idade $\left(r_{s}=0,350\right.$, p menor do que 0,01$)$ e com o tempo em diálise: $\left(r_{s}=0,320\right.$, p menor do que 0,05$)$. Inflamação avaliada pelo nível de proteína C-reativa ultrassensível foi significativamente associada à massa magra $\left(r_{s}=-0,283\right.$, p menor do que 0,05$)$.

Conclusões: $\mathrm{O}$ aumento do tempo em hemodiálise e a idade aumentada estiveram associados com pior estado nutricional. Nesta população, aparentemente quanto menor a massa magra corporal maior o nível de proteína C-reativa ultrassensível, sugerindo uma possível associação entre a inflamação e a massa magra corporal nestes pacientes.

DESCRITORES: DOENÇA RENAL CRÔNICA; HEMODIÁLISE; INFLAMAÇÃO; COMPOSIÇÃO CORPORAL.

\section{ABSTRACT}

Aims: To investigate the relationship of the inflammatory status with the lean body mass in hemodialysis patients.

Methods: A cross-sectional observational study included patients on hemodialysis for at least three months. The body composition analysis was performed by segmental multi-frequency bioimpedance (InBody $520^{\circledR}$ ). The nutritional status associated with inflammation was evaluated using the instrument that computes the Malnutrition-Inflammation Score.

Results: The sample included 59 subjects, 30 were female. The mean age was $58.7 \pm 14.4$ years, the median time on dialysis was 24 (9-49) months, the mean estimated dry weight was $67.0 \pm 14.7 \mathrm{~kg}$ and mean lean body mass was $29.7 \pm 5.5 \mathrm{~kg}$. The median serum level of ultrasensitive C-reactive protein was 8.6 (3.9 to 18.0$) \mathrm{mg} / \mathrm{L}$ and above the normal limit $(\leq 5.0 \mathrm{mg} / \mathrm{L})$, suggesting the presence of inflammation. The malnutrition-inflammation score had a median of 4 (2-6). There was a significant correlation between the score malnutrition-inflammation and age $\left(r_{s}=0.350, p\right.$ less than 0.01$)$ and with time on dialysis: $\left(r_{s}=0.320\right.$, $p$ less than 0.05$)$. Inflammation assessed by the level of ultrasensitive C-reactive protein was significantly associated with lean body mass $\left(\mathrm{r}_{\mathrm{s}}=-0.283\right.$, $\mathrm{p}$ less than 0.05$)$.

Conclusions: The increased time on dialysis and increased age were associated with poorer nutritional status. In this population, apparently the lower lean body mass the higher level of ultrasensitive C-reactive protein, suggesting a possible association between inflammation and lean body mass in these patients.

KEY WORDS: CHRONIC KIDNEY DISEASE; HEMODIALYSIS; INFLAMMATION; BODY COMPOSITION. 


\section{INTRODUÇÃO}

A doença renal crônica (DRC) é um problema de saúde pública mundial. Com a progressão da lesão renal, desenvolve-se um característico quadro clínico, que inclui anemia, acidose, anorexia, desnutrição proteico-calórica, elevação da pressão arterial, hipervolemia, hiperfosfatemia, hipercalemia, osteodistrofia renal e hiperparatireoidismo. ${ }^{1,2}$

A prevalência de desnutrição na DRC é elevada, afetando de 30 a $70 \%$ dos pacientes, dependendo do parâmetro utilizado, e contribui para o aumento do risco de morte e de piora da qualidade de vida. ${ }^{3}$ Em pacientes com DRC, a origem da relação inflamaçãodesnutrição é multifatorial. Distúrbios metabólicos, processo dialítico e anorexia têm envolvimento, induzindo à perda de proteínas corporais e à liberação de citocinas pró-inflamatórias que interferem neste processo. ${ }^{4,5} \mathrm{~A}$ desnutrição desenvolve-se naturalmente com a progressão da DRC, sendo um componente inerente da doença avançada. ${ }^{6}$

A ocorrência de inflamação, presente em indivíduos em hemodiálise (HD), está associada à obesidade, mas pode ter outras causas, entre as quais o aumento do catabolismo presente em pacientes com DRC. ${ }^{7,8}$ Nestes, a associação de desnutrição com inflamação tende a ser mais grave, sendo associada com o aumento da morbimortalidade em HD. ${ }^{9,10,11}$

O presente estudo tem por objetivo investigar a relação da inflamação com a massa magra corporal nos pacientes em HD.

\section{MÉTODOS}

O estudo, de caráter transversal e observacional, foi conduzido no Serviço de Nefrologia do Hospital São Lucas da Pontifícia Universidade do Rio Grande do Sul, com aprovação do Comitê de Ética e Pesquisa da instituição, sob registro número 08/04431. Foram incluídos na investigação pacientes em HD, clinicamente estáveis (não hospitalizados, sem infecção ativa e com acesso por fístula arteriovenosa) há no mínimo três meses, e que consentiram em participar do estudo pela assinatura do Termo de Consentimento Livre e Esclarecido. Todos os pacientes incluídos foram mantidos com a mesma prescrição de diálise: quatro horas, três vezes por semana, em máquinas Fresenius ${ }^{\circledR}$ 4008 S (Fresenius Medical Care, Bad Homburg, Alemanha), dialisador F80 (Fresenius Medical Care, São Paulo, Brasil), com heparina contínua, fluxo arterial entre $250-300 \mathrm{~mL} / \mathrm{minuto}$, fluxo de diali- sado (Fresenius Medical Care, São Paulo, Brasil) de $500 \mathrm{~mL} /$ minuto (usando bicarbonato como alcalinizante).

A avaliação nutricional foi realizada por medidas antropométricas, 30 minutos após o término da sessão de HD. Para tal mensuramos peso pós-diálise e altura, aferida por estadiômetro fixo à parede (Tonelli ${ }^{\circledR}$ ), com amplitude de medida de 400 a $2200 \mathrm{~mm}$ e escala em milímetros. A análise da composição corporal foi realizada por bioimpedância, utilizando aparelho InBody $520^{\circledR}$ (Biospace Co, Seul, Coreia do Sul), que realiza medição direta, segmentar, multifrequência $(5 \mathrm{KHz}, 50 \mathrm{KHz}$ e $500 \mathrm{KHz})$, com sistema tetrapolar de eletrodos, com oito pontos táteis, sendo dois em cada pé e dois em cada mão. Os pacientes receberam orientação quanto ao preparo necessário para fazer o exame na semana anterior. $O$ peso foi aferido no momento em que o paciente se posicionou na plataforma do aparelho de bioimpedância. Nesta análise de composição corporal são avaliados os componentes corporais: massa de gordura, magra e óssea e líquido corporal total. $\mathrm{O}$ índice de massa corporal (IMC) foi expresso em $\mathrm{kg} / \mathrm{m}^{2}$ e classificado conforme proposto pela Organização Mundial da Saúde, que para avaliação nutricional de idosos considera o mesmo ponto de corte do adulto. ${ }^{12}$

$\mathrm{O}$ estado nutricional relacionado à inflamação foi avaliado segundo um instrumento que computa o Escore Desnutrição-Inflamação (MIS - MalnutritionInflammation Score), que inclui critérios subjetivos e objetivos, é abrangente, é considerado um método superior à Avaliação Subjetiva Global ou medidas laboratoriais isoladas e tem se tornado um preditor prognóstico útil. O questionário possui 10 componentes, referentes à história clínica, ao exame físico, ao índice de massa corporal e a parâmetros laboratoriais. ${ }^{13,14} \mathrm{Cada}$ componente possui quatro níveis de severidade, de 0 (normal) a 3 (muito severo). $O$ escore final varia de 0 a 30 , denotando um grau crescente de severidade. Uma pontuação $\leq 8$ indica um estado nutricional normal ou desnutrição leve, 9-18 indica desnutrição moderada, e $>18$ indica desnutrição severa. ${ }^{15} \mathrm{O}$ nível inflamatório foi medido por proteína C-reativa ultrassensível (PCR-us) plasmática. Não foram utilizados outros marcadores em razão de não serem exames de rotina no serviço em que o estudo foi realizado. A coleta sanguínea foi realizada na primeira semana do mês, na segunda sessão de HD. A PCR-us foi quantificada por imunonefelometria de alta sensibilidade (Vitros ${ }^{\circledR}$ 5,1 FS, Ortho Clinical Diagnostic, Rochester, NY, EUA).

Para a análise dos dados utilizou-se o programa estatístico SPSS (Statistical Package for Social 
Sciences) versão 17.0. As variáveis contínuas são apresentadas como média e desvio padrão (DP) quando de distribuição simétrica, ou mediana e intervalo interquartil (IIQ) quando de distribuição assimétrica. As variáveis categóricas são apresentadas como frequência e porcentagem. Os testes de correlação de Pearson e o coeficiente de correlação de Spearman foram utilizados para avaliar as associações entre variáveis. O nível de significância foi definido como $\mathrm{p} \leq 0,05$.

\section{RESULTADOS}

A amostra foi constituída por 59 indivíduos, com uma distribuição homogênea no que diz respeito a sexo, sendo 30 do sexo feminino, com média de idade de 58,7 $( \pm 14,4)$ anos, composta por 27 adultos e 32 idosos e tempo mediano em HD de 24 (9-49) meses. Características da amostra estão descritas na Tabela 1.

Tabela 1. Características da amostra de pacientes em hemodiálise há pelo menos três meses, clinicamente estáveis, não hospitalizados. $(\mathrm{n}=59)$

\begin{tabular}{lc}
\multicolumn{1}{c}{ Variável } & Valor \\
\hline Idade (anos)* & $59(14)$ \\
\hline Sexo feminino: $\mathrm{n}(\%)$ & $30(51)$ \\
\hline $\begin{array}{l}\text { Principais comorbidades: } \mathrm{n}(\%) \\
\text { Hipertensão arterial }\end{array}$ & $33(56)$ \\
Doença cardíaca & $20(34)$ \\
Diabetes mellitus & $14(24)$ \\
Hiperparatireoidismo secundário & $11(19)$ \\
Tempo de diálise (meses) & \\
\hline
\end{tabular}

* Média \pm desvio padrão; ${ }^{\dagger}$ mediana (intervalo interquartil).

A média do IMC foi $25,0( \pm 4,8) \mathrm{kg} / \mathrm{m}^{2}$, ficando fora do limite da normalidade $\left(18,5\right.$ a $\left.24,9 \mathrm{~kg} / \mathrm{m}^{2}\right)$, na categoria de sobrepeso. A mediana do MIS foi 4,0 (2,0-6,0), considerado um estado nutricional normal ou desnutrição leve. A mediana do nível sérico de PCR-us foi 8,6 (3,9-18,0) $\mathrm{mg} / \mathrm{L}$ e acima do limite normal $(\leq 5,0 \mathrm{mg} / \mathrm{L})$, sugerindo a presença de inflamação. Não há definição de padrões de normalidade para classificação de massa magra, nesta população, assim, foi tomada por base a classificação fornecida pelo próprio exame de bioimpedância, considerando individualmente cada paciente. Os dados relativos às medidas antropométricas e de composição corporal são apresentados na Tabela 2.
Tabela 2. Medidas antropométricas/Composição corporal de pacientes em hemodiálise há pelo menos três meses, clinicamente estáveis, não hospitalizados. $(n=59)$

\begin{tabular}{|lc|}
\hline \multicolumn{1}{c}{ Variável } & Valor \\
\hline Peso seco $(\mathrm{kg})^{*}$ & $67,0(14,7)$ \\
\hline Massa magra $(\mathrm{kg})^{*}$ & $29,7(5,5)$ \\
\hline Índice de massa corporal $\left(\mathrm{kg} / \mathrm{m}^{2}\right)^{*}$ & $25,0(4,8)$ \\
\hline Proteína C-reativa ultrassensível $(\mathrm{mg} / \mathrm{L})^{+}$ & $8,6(3,9-18,0)$ \\
MIS $^{+}$ & $4,0(2,0-6,0)$ \\
\hline
\end{tabular}

MIS: Malnutrition-Inflammation Score (Escore Desnutrição-Inflamação). * Média \pm desvio padrão; ${ }^{\dagger}$ mediana (intervalo interquartil).

Nas correlações de Spearman houve associação positiva e moderada entre MIS e idade $\left(r_{s}=0,350\right.$, $\mathrm{p}<0,01)$ e entre MIS e tempo em HD $\left(\mathrm{r}_{\mathrm{s}}=0,320, \mathrm{p}<0,05\right)$. Inflamação, analisada pela PCR-us, foi negativamente associada à massa magra corporal $\left(\mathrm{r}_{\mathrm{s}}=-0,283\right.$, $\mathrm{p}<0,05)$.

\section{DISCUSSÃO}

Os dados sugerem que quanto maior o tempo em tratamento dialítico e mais avançada a idade do paciente, maior foi a pontuação no MIS, assinalando um pior estado nutricional. Desse modo, idade mais elevada e maior tempo em HD podem se associar à inflamação subclínica e a redução da síntese proteica. Sendo assim, a correlação positiva entre idade e MIS sugere redução do metabolismo corporal com o avançar da idade, não necessariamente dependente da presença de DRC ou de o indivíduo estar em HD.

Estudos prévios enfatizaram a relevância da desnutrição e da inflamação na DRC terminal e suas relações com risco aumentado de morte. ${ }^{17,18}$ Em coorte americana, pacientes em HD foram acompanhados por cinco anos com avaliação pelo MIS, associando-o com estado nutricional e sugerindo que ele possa ser eficaz na identificação de pacientes com risco de desnutrição. Os autores propõem que o MIS substitua os níveis de interleucina-6 e de PCR na clínica diária para avaliar o estado inflamatório. ${ }^{13} \mathrm{O}$ MIS encontrado na amostra atual é semelhante ao de estudo anterior. ${ }^{19} \mathrm{Na}$ Turquia, Demir et al. ${ }^{20}$ encontraram média de MIS $=6,0$, que se correlacionou positivamente com PCR-us $\left(r_{s}=0,31\right.$; $\mathrm{p}=0,01)$ e negativamente com albumina sérica $(\mathrm{r}=-0,42$; $\mathrm{p}<0,01) .{ }^{20}$ Estes dados reforçam a aplicabilidade do MIS como ferramenta clínica na predição do estado inflamatório em HD. Porém, o valor do MIS na análise atual foi bastante abaixo ao observado em estudo multicêntrico europeu (Dialysis Outcomes and Practice Patterns Study), em que o MIS variou entre 9,0 e 10,7, 
indicando desnutrição-inflamação mais avançada, quando comparado aos resultados do presente estudo, sugerindo pior estado de nutrição, associado à inflamação, em países europeus. ${ }^{21}$ Essas diferenças podem ser justificadas pela diversidade das populações em estudo, aos protocolos de tratamento dialítico, ou pela condição clínica dos pacientes avaliados.

O nível sérico de PCR-us foi bastante elevado, indicando a prevalência de inflamação na amostra. Estudos anteriores relacionaram marcadores séricos inflamatórios, baixos níveis de albumina sérica e MIS alto ao aumento da mortalidade em pacientes com doença renal crônica terminal. ${ }^{13,22}$ Há indicação de prognóstico pior na DRC, quando há associação de desnutrição com inflamação, com hipoalbuminemia, o que foi analisado por Danielski et al., sugerindo que níveis de marcadores inflamatórios e de estresse oxidativo apresentavam-se elevados em pacientes que tinham hipoalbuminemia. ${ }^{24}$ De Mutsert et al. ${ }^{16}$ justificaram o mais alto risco de mortalidade (47\%) associado à redução do nível sérico de albumina $(1 \mathrm{~g} / \mathrm{dL})$, pela presença de inflamação. ${ }^{16}$ Assim, pacientes que apresentam DRC devem passar por tratamento com nutricionistas para suprir suas necessidades de micronutrientes que são perdidos na DRC. Adquirindo, dessa maneira, uma dieta de qualidade, pode haver uma redução significativa na inflamação e uma melhora na produção energética e proteica. ${ }^{25}$

Embora a massa magra corporal, na amostra atual, estivesse dentro da realidade, os resultados corroboraram dados anteriores que evidenciaram a inflamação como possível responsável pelos efeitos negativos à massa magra corporal de pacientes em HD. Entretanto, não fizemos a análise de sobrevida da amostra para poder relacionar esses dados com mortalidade. Logo, um estudo mais amplo com um maior número de pacientes e com a análise da sobrevida faz-se necessário para que possamos compreender ainda mais a relação da inflamação com os efeitos danosos à massa magra corporal de pacientes em HD e buscar um melhor tratamento e conforto aos pacientes.

A desnutrição proteico-calórica é um fator que reduz a qualidade de vida e piora o prognóstico dos pacientes com DRC, pois as perdas de massa muscular e de gordura se relacionam com a mortalidade de pacientes em HD. ${ }^{26}$ Há grande variabilidade nos dados de nutrição em pacientes com DRC, pela utilização de diferentes instrumentos usados no diagnóstico nutricional. ${ }^{27,28,29}$ Além disso, o paciente com DRC apresenta significativas diferenças em sua composição corporal..$^{30}$ Quando não detectadas, essas diferenças podem influenciar no diagnóstico e no prognóstico do paciente. A utilização da bioimpedância segmentar multi-frequência vem crescendo e parece ser uma ferramenta clinicamente importante para a detecção precoce de alterações da composição corporal e para a monitorização do estado nutricional. ${ }^{27,31}$

A despeito de já se ter sugerido que o tempo em diálise possa ser um fator negativo sobre o peso e a composição corporal, não encontramos relação entre o tempo em tratamento dialítico e os parâmetros antropométricos utilizados. ${ }^{32}$ Os marcadores bioquímicos e nutricionais podem sofrer variações, dependentes de fatores como: tempo em diálise, presença de infecção aguda, perda do acesso vascular, ou outros fatores interferentes. Entretanto, não houve a ocorrência de qualquer evento agudo perturbador, durante o período de observação.

Sumarizando, os resultados atuais mostram que maior tempo em HD e idade aumentada estiveram associados com pior estado nutricional. Nesta população, aparentemente quanto menor a massa magra corporal maior o nível de PCR-us, sugerindo uma possível associação entre a inflamação e a massa magra corporal nestes pacientes.

\section{REFERÊNCIAS}

1. National Kidney Foundation: K/DOQI Clinical Practice Guidelines for Chronic Kidney Disease: evaluation, classification, and stratification. Am J Kidney Dis 2002;39(2 Suppl 1):S1-266.

2. Moranne O, Froissart M, Rossert J, Gauci C, Boffa JJ, Haymann JP, M'rad MB, Jacquot C, Houillier P, Stengel B, Fouqueray B and The NephroTest Study Group. Timing of onset of CKD-related metabolic complications. J Am Soc Nephrol 2009;20(1):164-71.

3. Dukkipati R, Kopple JD. Causes and prevention of protein-energy wasting in chronic kidney failure. Semin Nephrol. 2009;29(1): 39-49.

4. Fouque D, Pelletier S, Mafra D, Chauveau P. Nutrition and Chronic Kidney Disease. Kidney Int. 2011;80(4):348-57.

5. Aatif T, Hassani K, Alayoud A, Maoujoud O, Ahid S, Benyahia M, Oualim Z. Parameters to Assess Nutritional Status in a Moroccan Hemodialysis Cohort. Arab J of Nephrol and Transplant. 2013;6(2):89-97.

6. Mekki K, Remaoun M, Belleville J, Bouchenak M. Hemodialysis duration impairs food intake and nutritional parameters in chronic kidney disease patients. Int Urol Nephrol. 2012;44(1):237-44. 
7. Ikizler TA. Nutrition, inflammation and chronic kidney disease. Cur Opin Nephrol Hypertens. 2008;17(2):162-167.

8. Beberashvili I, Sinuani I, Azar A, Yasur H, Feldman L, Efrati S, Averbukh Z, Weissgarten J. Nutritional and inflammatory status of hemodialysis patients in relation to their body mass index. J Ren Nutr. 2009;19(3):238-47.

9. Ibrahim S, El Salamony O. Depression, quality of life and malnutrition-inflammation scores in hemodialysis patients. Am J Nephrol. 2008;28(5):784-91.

10. Kalantar-Zadeh K, Block G, McAllister CJ, Humphreys MH, Kopple JD. Appetite and inflammation, nutrition, anemia, and clinical outcome in hemodialysis patients. Am J Clin Nutr. 2004;80(2):299-307.

11. Toledo FR, Antunes AA, Vannini FC, Silveira LV, Martin LC, Barretti P, Caramori JC. Validity of malnutrition scores for predicting mortality in chronic hemodialysis patients. Int Urol Nephrol. 2013. DOI: 10.1007/s11255-013-0482-3

12. World Health Organization. Physical status: the use and interpretation of anthropometry. Geneva: WHO Technical Report Series, n. 854; 1995. Disponível em: <http://whqlibdoc.who.int/trs/WHO_TRS_854.pdf>.

13. Rambod M, Bross R, Zitterkoph J, Benner D, Pithia J, Colman S, Kovesdy CP, Kopple JD, Kalantar-Zadeh K. Association of MalnutritionInflammation Score with quality of life and mortality in hemodialysis patients: a 5-year prospective cohort study. Am J Kidney Dis 2009;53(2):298-309.

14. Pisetkul C, Chanchairujira K, Chotipanvittayakul N, Ong-Ajyooth L, Chanchairujira T. Malnutrition-inflammationscore associated with atherosclerosis, inflammation and short-term outcome in hemodialysispatients. J Med Assoc Thai. 2010; 93 Suppl 1:S147-56.

15. Kalantar-Zadeh K, Kopple JD, Block G, Humphreys MH. A malnutrition-inflammation score is correlated with morbidity and mortality in maintenance hemodialysis patients. Am J Kidney Dis. 2001;38(6):1251-63.

16. De Mutsert R, Grootendorst DC, Indemans F, Boeschoten EW, Krediet RT, Dekker FW for the Nederlands Cooperative Study on Adequacy of Dialysis-II Study Group. Association between serum albumin and mortality in dialysis patients is partly explained by inflammation, and not by malnutrition. J Ren Nutr. 2009;19(2):127-35.

17. Fiedler R, Jehle PM, Osten B, Dorligschaw O, Girndt M. Clinical nutrition scores are superior for the prognosis of haemodialysis patients compared to lab markers and bioelectrical impedance. Nephrol Dial Transplant. 2009;24(12):3812-7.

18. Ho LC, Wang HH, Peng YS, Chiang CK, Huang JW, Hung KY, Hu FC, Wu KD. Clinical utility of malnutrition-inflammationscore in maintenance hemodialysispatients: focus on identifying the best cut-off point. Am J Nephrol. 2008;28(5):840-6.

19. Rattanasompattikul M, Molnar MZ, Zaritsky JJ, Hatamizadeh P, Jing J, Norris KC, Kovesdy CP, Kalantar-Zadeh K. Association of malnutrition-inflammation complex and responsiveness to erythropoiesis-stimulating agents in long-term hemodialysis patients. Nephrol Dial Transplant. 2013;28(7):1936-45.

20. Demir M, Kucuk A, Sezer MT, Altuntas A, Kaya S. Malnutrition-inflammationscore and endothelial dysfunction in hemodialysis patients. J Ren Nutr. 2010;20(6):377-83.

21. Combe C, McCullough KP, Asano Y, Ginsberg N, Maroni BJ, Pifer TB. Kidney Disease Outcomes Quality Initiative (K/DOQI) and the Dialysis Outcomes and Practice Patterns Study (DOPPS): nutrition guidelines, indicators, and practices. Am J Kidney Dis. 2004;44 (5 Suppl 2):39-46.

22. Colman S, Bross R, Benner D, Chow J, Braglia A, Arzaghi J, Dennis J, Martinez L, Baldo DB, Agarwal V, Trundnowski T, Zitterkoph J, Martinez B, Khawar OS, Kalantar-Zadeh K. The nutritional and inflammatory evaluation in dialysis patients (NIED) study: overview of the NIED study and the role of dietitians. J Ren Nutr. 2005;15(2):231-43.

23. Jialal I, Devaraj S, Venugopal SK. C-reactive protein: risk marker or mediator in atherothrombosis? Hypertension. 2004;44(1):6-11.

24. Danielski M, Ikizler TA, McMonagle E et al. Linkage of hypoalbuminemia, inflammation, and oxidative stress in patients receiving maintenance hemodialysis therapy. Am J Kidney Dis. 2003; 42:286-94.

25. Steiber AL. Chronic Kidney Disease: Considerations for Nutrition Interventions. JPEN J Parenter Enteral Nutr 2014. DOI $10.1177 / 0148607114527315$

26. Huang CX, Tighiouart H, Beddhu S, Cheung AK, Dwyer JT, Eknoyan G, Beck GJ, Levey AS, Sarnak MJ. Both low muscle mass and low fat are associated with higher allcause mortality in hemodialysis patients. Kidney Int. 2010;77(7):624-9.

27. Bellizzi V, Scalfi L, Terracciano V, De Nicola L, Minutolo R, Marra M. et al. Early changes in bioelectrical estimates of body composition in chronic kidney disease. J Am Soc Nephrol. 2006;17(5):1481-7.

28. De Oliveira C, Kubrusly M, Mota RS, Da Silva CAB, Oliveira VN. Desnutrição na insuficiência renal crônica: qual o melhor método diagnóstico na prática clínica? J Bras Nefrol. 2010;32(1):57-70.

29. Canpolat N, Caliskan S, Sever L, Tasdemir M, Ekmekci OB, Pehlivan G, Shroff R. Malnutrition and its association with inflammation and vascular disease in children on maintenance dialysis. Int Urol Nephrol. 2013. DOI: 10.1007/s00467-013-2527-3

30. Piratelli CM, Telarolli Junior R. Nutritional evaluation of stage 5 chronic kidney disease patients on dialysis. Sao Paulo Med J. 2012;130(6):392-7.

31. Macdonald JH, Marcora SM, Jibani M, Roberts G, Kumwenda MJ, Glover R. et al. Bioelectrical impedance can be used to predict muscle mass and hence improve estimation of glomerular filtration rate in non-diabetic patients with chronic kidney disease. Nephrol Dial Transplant. 2006;21(12):3481-7.

32. Chertow GM, Johansen KL, Lew N, Lazarus JM, Lowrie EG. Vintage, nutritional status, and survival in hemodialysis patients. Kidney Int. 2000; 57(3):1176-81. 